\title{
Nonintubated video-assisted thoracoscopic surgery using adaptive servo ventilation in a patient with severe respiratory dysfunction: a case report
}

\author{
Yuki Kikuchi ${ }^{1 *}$, Masaki Orihara ${ }^{2}$, Rie Mieda ${ }^{2}$ and Shigeru Saito ${ }^{2}$
}

\begin{abstract}
Background: Video-assisted thoracoscopic surgery (VATS) is usually performed under general anesthesia with a double-lumen tube. Recently, VATS without tracheal intubation in a patient with severe respiratory dysfunction has been reported. A case of nonintubated (also known as awake or tubeless) VATS using adaptive servo ventilation (ASV), a form of noninvasive positive pressure ventilation providing varying amounts of ventilator support, is presented. This is the first report of nonintubated VATS using ASV.

Case presentation: A 60-year-old woman was scheduled for VATS bullectomy for the treatment of pneumothorax. She had severe respiratory dysfunction and had been receiving ASV therapy because of type 2 respiratory failure. Thus, nonintubated VATS using ASV, epidural anesthesia, and dexmedetomidine were selected. When surgical pneumothorax was created by incision of the pleura, her respiratory status remained stable. In addition, lung collapse was easily induced at operation. The leaking bulla was easy to identify, and bullectomy was performed. During surgery, she continued spontaneous breathing and did not complain of pain or discomfort. She was transferred to the intensive care unit with ASV and discharged on postoperative day 12 with no respiratory complications.

Conclusion: It is necessary to maintain a stable respiratory status, as well as adequate analgesia and sedation, during nonintubated VATS in patients with severe respiratory dysfunction. When total lung collapse is not necessary for the surgical procedure, use of ASV would be an effective strategy.
\end{abstract}

Keywords: Nonintubated video-assisted thoracoscopic surgery, Adaptive servo ventilation, Respiratory dysfunction, Epidural anesthesia, Dexmedetomidine

\section{Background}

Video-assisted thoracoscopic surgery (VATS) is usually performed under general anesthesia with a double-lumen tube to achieve single-lung ventilation [1]. However, general anesthesia and tracheal intubation carry high risks for patients with various thoracic diseases, including those with respiratory dysfunction. Recently, VATS without tracheal intubation has been reported to be feasible and safe for patients with respiratory dysfunction [1-5]. VATS without tracheal intubation is distinct from conventional

\footnotetext{
* Correspondence: y.iszmi8711@gmail.com

${ }^{1}$ Department of Anesthesiology, Saiseikai Utsunomiya Hospital, 911-1

Takebayashi-machi, Utsunomiya-shi, Tochigi 321-0974, Japan

Full list of author information is available at the end of the article
}

general anesthesia with tracheal intubation because patients breathe spontaneously. However, spontaneously breathing patients' oxygenation and ventilation can be impaired because of a surgical pneumothorax [2]. Therefore, careful respiratory management is essential to maintain the physiological status during VATS without tracheal intubation.

Adaptive servo ventilation (ASV) is a form of noninvasive positive pressure ventilation (NPPV) [6]. It provides a background level of expiratory positive airway pressure (EPAP) to which a variable amount of inspiratory pressure support (IPAP) is added. The pressure support (IPAP-EPAP) applied varies depending on the subjects' ventilatory effort [7]. 


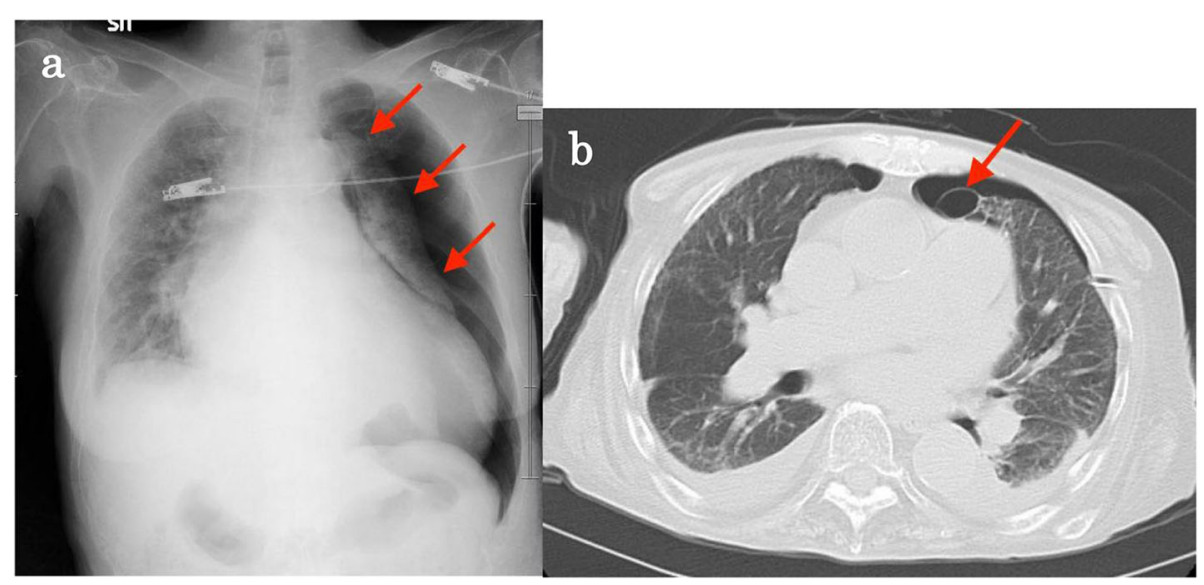

Fig. 1 Preoperative images of the patient. a Chest X-ray shows the collapsed lung (red arrows). The left pneumothorax is severe. $\mathbf{b}$ Computed tomography shows a bulla of the left upper lobe behind the sternum (red arrow). The bulla was suspected as the cause of the pneumothorax.

A case of nonintubated (also known as awake or tubeless) VATS bullectomy for the treatment of pneumothorax using ASV, epidural anesthesia, and dexmedetomidine in a patient with severe respiratory dysfunction is presented. This is the first report of nonintubated VATS using ASV.

\section{Case presentation}

A 60-year-old woman (height $154 \mathrm{~cm}$, weight $35 \mathrm{~kg}$ ) was transported to our hospital by ambulance with chest pain and dyspnea. After evaluation, she was diagnosed with left pneumothorax (Fig. 1). She had a history of muscular dystrophy, dilated cardiomyopathy, and atrial fibrillation. She had been receiving ASV therapy for 2 years because of type 2 respiratory failure. Before admission, she was classified as Hugh-Jones level 5 with the oxygen flow rate, IPAP, and EPAP set to $1 \mathrm{~L} / \mathrm{min}, 7-16 \mathrm{~cm} \mathrm{H}_{2} \mathrm{O}$, and $4 \mathrm{~cm} \mathrm{H}_{2} \mathrm{O}$, respectively. Prehospital respiratory function test and transthoracic echocardiography results are presented in Table 1. Despite chest tube drainage and pleurodesis, the air leak persisted. Thus, VATS bullectomy was

Table 1 Prehospital respiratory function test and transthoracic echocardiography results

\begin{tabular}{ll}
\hline Respiratory function test & \\
\hline Vital capacity (L) & 0.89 \\
\hline$\%$ vital capacity (\%) & 32.80 \\
Forced expiratory volume in one second (L) & 0.82 \\
Forced expiratory volume \% in one second (\%) & 93.18 \\
Transthoracic echocardiography & \\
Left ventricular end-diastolic diameter (mm) & 47 \\
Left ventricular end-systolic diameter (mm) & 40 \\
Ejection fraction (\%) & 35 \\
Tricuspid regurgitation & Moderate \\
Mitral regurgitation & Mild \\
\hline
\end{tabular}

scheduled. It was thought that the operation would not take much time because of the substernal location of the bulla suspected as the cause of the pneumothorax. Moreover, she had severe respiratory dysfunction and refused tracheal intubation due to the risk of tracheal intubation, and she also considered that initiation of mechanical ventilation would make it difficult to wean from mechanical ventilation. Therefore, VATS without tracheal intubation was chosen.

Standard monitoring included electrocardiogram, arterial blood pressure, and pulse oximetry. She was kept on ASV performed with a full face mask in the operating room. The settings were oxygen flow rate, $4 \mathrm{~L} / \mathrm{min}$; IPAP, $7-16 \mathrm{~cm} \mathrm{H}_{2} \mathrm{O}$; and EPAP, $4 \mathrm{~cm} \mathrm{H}_{2} \mathrm{O}$. After peripheral arterial cannulation, an epidural catheter was placed at the thoracic 5-6 level, and $3 \mathrm{ml}$ of $0.5 \%$ ropivacaine were injected through the epidural catheter following $2 \mathrm{ml}$ of $1 \%$ mepivacaine as a test dose. Then, an infusion of $1 \mu \mathrm{g} / \mathrm{kg} / \mathrm{h}$ dexmedetomidine was started. Before she was turned to the right lateral position, it was confirmed that the extent of epidural anesthesia was from thoracic 2 to 8 . Local anesthesia was not performed. A 5-cm skin incision was made in the third intercostal space. When surgical pneumothorax was created by incision of the pleura, her respiratory status remained stable. In addition, lung collapse was obtained with ease at operation while she was on ASV, a form of NPPV. In the middle of the operation, the dosage of dexmedetomidine was reduced to $0.6 \mu \mathrm{g} / \mathrm{kg} / \mathrm{h}$ to maintain the Richmond Agitation-Sedation Scale around -2 . The leaking bulla was easily identified, and bullectomy was performed. After a negative air leak test, a chest tube was inserted. The operation time was $56 \mathrm{~min}$, and the anesthesia time was $116 \mathrm{~min}$. During surgery, although her arterial blood gas analysis showed respiratory acidosis (Table 2), oxygen saturation was maintained 
Table 2 Preoperative and intraoperative arterial blood gas data

\begin{tabular}{llll}
\hline & Preoperation & At the time of operation & End of operation \\
\hline $\mathrm{pH}$ & 7.421 & 7.366 & 7.386 \\
$\mathrm{PaCO}_{2}(\mathrm{mmHg})$ & 45.3 & 53.4 & 50.8 \\
$\mathrm{PaO}_{2}(\mathrm{mmHg})$ & 123.0 & 125.0 & 154.5 \\
Base excess $(\mathrm{mEq} / \mathrm{L})$ & 4.6 & 4.8 & 5.0 \\
\hline
\end{tabular}

higher than 90\%. In addition, she continued spontaneous breathing and did not complain of pain or discomfort. She was transferred to the intensive care unit (ICU) with ASV. The chest tube was removed on postoperative day (POD) 1 , and she was transferred out of the ICU on POD 2. She was discharged on POD 12 with no respiratory complications.

\section{Discussion}

A case of nonintubated VATS using ASV was described. Our experience indicates that ASV can be successfully used to maintain a stable respiratory status during nonintubated VATS with severe respiratory dysfunction, in combination with epidural anesthesia and dexmedetomidine.

Nonintubated VATS was first reported for diagnostic procedures in 1979 [5]. In recent years, nonintubated VATS strategies are becoming increasingly used worldwide [4]. However, there is always the possibility of the need to convert to general anesthesia with tracheal intubation, although complications and adverse effects following general anesthesia, tracheal intubation, and one-lung ventilation are inevitable. To prevent conversion to general anesthesia, stable spontaneous breathing following adequate analgesia and sedation is required [3].

In the present case, the patient had used ASV before admission. Thus, it was decided that the patient would continue on ASV in the operating room. Because ASV is a form of NPPV, the operated lung did not collapse completely during the surgical pneumothorax. With surgically induced open pneumothorax, the operated lung usually collapses progressively. The dependent lung is then responsible for sufficient respiratory function, including oxygenation and ventilation [2]. That would not allow the patient with severe respiratory dysfunction to achieve stable respiration. The present patient did not need total lung collapse because of the substernal location of the bulla suspected as the cause of her pneumothorax. If total lung collapse is not necessary, use of ASV would be an effective strategy during nonintubated VATS.

It is essential to provide adequate analgesia and sedation in addition to careful respiratory management. Various approaches to analgesia have been developed and proven feasible, including the current mainstream of thoracic epidural anesthesia, paravertebral nerve block, and percutaneous or thoracoscopic intercostal nerve block [8]. Moreover, various drugs such as propofol, midazolam, and dexmedetomidine have been used as sedatives $[3,8]$. Dexmedetomidine provides an anxiolytic effect and cooperative sedation without respiratory depression [9]. Thus, dexmedetomidine may be a suitable sedative for nonintubated VATS.

\section{Conclusion}

It is necessary to maintain a stable respiratory status during nonintubated VATS in patients with severe respiratory dysfunction. When total lung collapse is not necessary for the surgical procedure, use of ASV would be an effective strategy.

\section{Abbreviations}

ASV: Adaptive servo ventilation; EPAP: Expiratory positive airway pressure; ICU: Intensive care unit; IPAP: Inspiratory positive airway pressure; NPPV: Noninvasive positive pressure ventilation; POD: Postoperative day; VATS: Video-assisted thoracoscopic surgery

\section{Acknowledgements}

Not applicable

Authors' contributions

YK and MO wrote the manuscript. RM and SS revised and edited the manuscript. All authors read and approved the final manuscript.

Funding

Not applicable

Availability of data and materials

Data relevant to this case report are not available for public access because of patient privacy concerns but are available from the corresponding author on reasonable request.

Ethics approval and consent to participate Not applicable

Consent for publication

Written, informed consent for publication was obtained from the patient using a form approved by the ethics committee of Gunma University Hospital.

\section{Competing interests}

The authors declare that they have no competing interests.

\section{Author details}

'Department of Anesthesiology, Saiseikai Utsunomiya Hospital, 911-1 Takebayashi-machi, Utsunomiya-shi, Tochigi 321-0974, Japan. ${ }^{2}$ Department of Anesthesiology, Gunma University Graduate School of Medicine, 3-39-22 Showa-machi, Maebash-shi, Gunma 371-8511, Japan. 
Received: 22 July 2019 Accepted: 28 August 2019

Published online: 03 September 2019

\section{References}

1. Hung MH, Hsu HH, Cheng YJ, Chen JS. Nonintubated thoracoscopic surgery: state of the art and future directions. J Thorac Dis. 2014;6(1):2-9.

2. Liu YJ, Hung MH, Hsu HH, Chen JS, Cheng YJ. Effects on respiration of nonintubated anesthesia in thoracoscopic surgery under spontaneous ventilation. Ann Transl Med. 2015;3(8):107.

3. Iwata Y, Hamai Y, Koyama T. Anesthetic management of nonintubated video-assisted thoracoscopic surgery using epidural anesthesia and dexmedetomidine in three patients with severe respiratory dysfunction. J Anesth. 2016;30(2):324-7.

4. Tacconi F, Pompeo E. Non-intubated video-assisted thoracic surgery: where does evidence stand? J Thorac Dis. 2016;8(Suppl 4):S364-75.

5. Zheng H, Hu XF, Jiang GN, Ding JA, Zhu YM. Nonintubated-awake anesthesia for uniportal video-assisted thoracic surgery procedures. Thorac Surg Clin. 2017;27(4):399-406.

6. Momomura S, Seino Y, Kihara Y, Adachi H, Yasumura Y, Yokoyama H. Adaptive servo-ventilation therapy using an innovative ventilator for patients with chronic heart failure: a real-world, multicenter, retrospective, observational study (SAVIOR-R). Heart Vessels. 2015;30(6):805-17.

7. Szollosi I, O'Driscoll DM, Dayer MJ, Coats AJ, Morrell MJ, Simonds AK. Adaptive servo-ventilation and deadspace: effects on central sleep apnoea. J Sleep Res. 2006;15(2):199-205.

8. Yang JT, Hung MH, Chen JS, Cheng YJ. Anesthetic consideration for nonintubated VATS. J Thorac Dis. 2014;6(1):10-13.

9. Eberl S, Preckel B, Bergman JJ, Hollmann MW. Safety and effectiveness using dexmedetomidine versus propofol TCI sedation during oesophagus interventions: a randomized trial. BMC Gastroenterol. 2013;13:176.

\section{Publisher's Note}

Springer Nature remains neutral with regard to jurisdictional claims in published maps and institutional affiliations.

\section{Submit your manuscript to a SpringerOpen ${ }^{\circ}$ journal and benefit from:}

- Convenient online submission

- Rigorous peer review

- Open access: articles freely available online

High visibility within the field

- Retaining the copyright to your article

Submit your next manuscript at $\boldsymbol{\nabla}$ springeropen.com 